\section{Degrowth als ein Gedanke der Nachhaltigkeit}

Bericht über die internationale Konferenz „Degrowth, Ecological Sustainability and Social Equity“

Venedig, Italien, 19.-23. September 2012

\section{von Julia Hahn, Linda Nierling und Michael Reuß, ITAS, sowie Petra Wächter, ITA Wien}

Was meint „Degrowth“? Es handelt sich um einen alternativen Ansatz zum ökonomischen Wachstumszwang, der in Industriestaaten mit der Vorstellung verbunden ist, der Wohlstand einer Gesellschaft zeige sich (ausschließlich) in deren wirtschaftlichem Wachsen. Es geht beim Degrowth-Ansatz damit auch um Nachhaltigkeitsthemen und die wachsende Abhängigkeit von Technologien.

Die Konferenz war stark durch den Versuch geprägt, Wissenschaft und Zivilgesellschaft näher zusammenzubringen. Dies zeigte sich zum einen in der Konzeption der inhaltlichen Beiträge, in denen Vorträge sowohl von WissenschaftlerInnen und zivilgesellschaftlichen AkteurInnen gehalten wurden, aber auch in der Ausgestaltung der Sessions selbst, die viel Zeit für Diskussion untereinander vorsahen. Zum anderen ging es den VeranstalterInnen darum, die Venezianische Bevölkerung einzubinden, was in der frei zugänglichen Eröffnungsveranstaltung im Theater Malibran mit über 1.000 BesucherInnen, den vielfältigen Parallelevents (öffentlichen Treffen und Debatten, $\mathrm{Mu}$ sik, Filmen und Buchpräsentationen) sowie der Vorstellung vieler Ergebnisse der Konferenz in der „Garden of Ideas“" sehr gut gelang. Zum Rahmenprogramm der Konferenz gehörte neben der Messe „Altrofuturo“, bei der regionale, biologisch produzierte Produkte angeboten wurden und Konzerte stattfanden, auch eine Filmschau. Mit ca. 700 TeilnehmerInnen aus über 35 Ländern in Venedig hatte sich die TeilnehmerInnenzahl gegenüber der ersten Konferenz 2008 in Paris und der zweiten in Barcelona 2010 mehr als verdoppelt.

Jeder Konferenztag hatte ein Hauptthema: „Commons“, „Democracy“ und „Work“ mit täglich mehr als 20 verschiedenen Workshops, die Vormittags durch eher wissenschaftliche Vorträge und nachmittags durch Diskussionen und Erarbeitung von Proposals gestaltet waren. Täglich fanden auch morgens und abends Plenary Sessions statt, bei denen einerseits bekannte DegrowthProponentInnen wie Serge Latouche, Filka Sekulova, Francois Schneider, Giorgos Kallis, Jean Martínez-Alier vertreten waren, anderseits aber auch VertreterInnen von vielen anderen nahen Disziplinen wie Ökofeminismus oder Klimatologie, Theologie, sowie Nicht-WissenschafterInnen, wie GewerkschafterInnen, UmweltaktivistInnen oder MetallarbeiterInnen zu Wort kamen.

\section{Inhaltliche Auseinandersetzungen}

Die gut besuchte Session „The Role of Technology in a Degrowth Society" wurde von Linda Nierling und Oliver Parodi (beide ITAS) geleitet. Die Vorträge behandelten das Thema aus sehr unterschiedlichen Perspektiven, was sich jedoch am Ende zu einem breiten Überblick zur bislang kaum behandelten Thematik zusammenfügte. Oliver Parodi führte in seinem Vortrag "Interdependencies of Technology and Degrowth. What has technology to do with Degrowth?" die kulturell-philosophischen Grundlagen von Degrowth aus und betonte den notwendigen grundlegenden Wandel im Verständnis, Umgang und in der Entwicklung von Technologie innerhalb von Degrowth. Die kulturelle Perspektive ermögliche ein Verstehen und eine Diskussion über die tiefen Einstellungen gegenüber Technologie und könne daher ein Gestalten einer anderen Technologie ermöglichen, die besser an die Bedürfnisse einer Degrowth-Gesellschaft angepasst seien. Mario Pansera (University of Exeter Business School) zeigte in seinem Vortrag „Eco-innovation as a Development Tool: Evidence from Latin America and Asia" anhand vieler Beispiele neue Wege auf, wie lokale Technologieentwicklung im globalen Süden ausgestaltet werden könne. Den Abschluss bildete der Beitrag ,Sustainable consumption and technological innovation in a Degrowth society" von Philip J. Vergragt (Tellus Institute, USA), der über die Beziehung zwischen Degrowth und Nachhaltigkeit und den gesellschaftlichen Gebrauch von Technologie in diesem Zusammenhang sprach. Um die vielfach geforderte Transformation der Gesellschaft anzugehen, schlug er 
den Ansatz des „transition managements“ vor, der mithilfe neuer Policy-Instrumente und der Schaffung von Nischen für neue Technologien Änderungen planbarer zu machen versucht.

Der zweite Teil des Workshops war der Diskussion gewidmet. Die Beiträge vom Morgen legten nahe, drei unterschiedliche Felder in den Blick zu nehmen: Zum einen sich mit der normativen Verfasstheit von Technologieentwicklung und -einsatz zu beschäftigen, zum zweiten das NordSüd-Verhältnis der Technologieentwicklung in den Blick zu nehmen und zum dritten, sich der Ausgestaltung bzw. den Problemen konkreter Technologien, wie den prominenten und allgegenwärtigen Informationstechnologien, zu widmen. Die Diskussionen zeigten v. a. auf, dass der Übergang in eine Degrowth-Gesellschaft ohne Technologien nicht gedacht werden kann. Konzeptionelle und praktische Überlegungen, wie dieser Prozess von statten geht, sind sehr notwendig, stehen bislang aber erst am Anfang. Der Workshop bildete einen ersten Aufschlag dafür, dieses Thema einzugrenzen und relevante Fragestellungen zu entwickeln, die - so ist zu hoffen - im Rahmen der Degrowthdebatte weiterverfolgt werden.

Im Workshop „Towards a society of the commons: from the democracy of the earth to new institutionalities" ging es um große technologische Veränderungen im Energiebereich, Dezentralisierung und Partizipation. Hierzu gab es von zwei der Vortragenden länderspezifische Fallbeispiele aus Italien und Deutschland. In seinem Vortrag betrachtete Luigi Gaudio (University College London) eine Genossenschaft in Süditalien, die Solarzellen bereitstellt und die lokale Bevölkerung einbezieht. Dieses Fallbeispiel ermöglichte ihm, das Potenzial von kleinteiligen, regionalen Initiativen für eine gesamtgesellschaftliche Transformation hin zu alternativen Energieformen zu untersuchen. Der Vortrag von Julia Hahn (ITAS) stellte den nationalen Bürgerdialog des BMBFs zum Thema Energietechnologien für die Zukunft vor. Dabei ging es darum, diesen Partizipationsprozess (mit einer relativ hohen Anzahl an Beteiligten) im Rahmen von „responsible innovation“ und Nachhaltigkeit kritisch zu betrachten, um die unterschiedlichen Erwartungshaltungen von BürgerInnen, ExpertInnen und PolitikerInnen, aber auch den Mehrwert von „guter“ Mitbestimmung zu erkennen. Die anschließenden Diskussionen zeigten u. a. die Schwierigkeiten, v. a. die Aufnahme von Ergebnissen dieser Prozesse in die Politik zu ermöglichen, umso auch eine Verlässlichkeit und Glaubwürdigkeit von partizipativen Verfahren zu gewährleisten.

In der Session zu „Land use, competing requirements and the role of spatial planning institutions" gab es nach dem Input von Petra Wächter (ITA Wien) einen Vortrag von Mario Agostinelli (Associazione Energiafelice) über die Rolle von Gemeinschaftsgütern für soziale Bewegungen und einen weiteren von Jerry Van den Berge (European Federation of Public Service Unions) zu einer EU-weiten Initiative gegen die Privatisierung von Wasser, bei dem die Problematik einer restriktiven Nutzung von Wasser im Mittelpunkt stand. Dies gab Anstoß zu weiteren Diskussionen über den öffentlichen Zugang zu natürlichen Ressourcen und darüber, inwiefern durch technische Mittel der Zugang erleichtert oder behindert werden kann.

Am Nachmittag wurden Pläne darüber geschmiedet, wie in einem kleinen italienischen Tal ein Stück Land, das zur öffentlichen Nutzung ausgeschrieben wurde und für das es noch keine InteressentInnen gibt, ein Projekt im Sinne von Degrowth aussehen könnte.

In der Postersession am zweiten Konferenztag war eine kleine aber bunte Mischung von Themen vertreten. Diese reichten von der Frage nach dem Nutzen der Wirtschaft an sich über Policy-Instrumente für eine nachhaltige Stadtentwicklung bis hin zu handfesten Projekten wie der Papierherstellung aus dem Abfall von Zitrusfrüchten und dem Bau einer kleinen Windturbine für den eigenen Strombedarf.

\section{Rahmenprogramm der Konferenz}

Zusätzlich zu der theoretischen Auseinandersetzung und Diskussion gab es während der Konferenz auch Möglichkeiten, praktische Erfahrungen auszutauschen. Die Themen der sog. „activity workshops“, die parallel zu den übrigen Veranstaltungen der Konferenz liefen, reichten von Yoga am Morgen über Themen wie Kunst aus Abfall, Achtsamkeit, Integration von Degrowth in die Schulbildung hin zu praktischen 
Anleitungen zum Bau einer Windturbine mit Hilfe einfacher Werkzeuge und Materialien. So lieferte der Workshop „How to construct a small wind turbine using simple tools, materials and techniques" nicht nur praktische Anleitungen dafür, wie man aus Holz und Metall in $600 \mathrm{Ar}$ beitsstunden eine Windturbine zur Deckung des eigenen Strombedarfs bauen kann. Der Workshop zeigte auch auf, dass es neben der bekannten „Open Source Software“- auch eine „Open Source Hardware"-Bewegung gibt, im Rahmen derer technische Anleitungen im Detail ausgearbeitet und im Internet bereitgestellt werden. Dies ermöglicht die lokale Produktion von hoch komplexen technischen Geräten mit einfachen Mitteln auf lokaler Ebene.

Die begleitende Filmschau veranschaulichte, wie sich ein solches Konzept in individuellen Lebensentwürfen und im politischen Leben niederschlagen kann. Die Filme zeigten zum einen, in welcher Weise das Degrowth-Paradigma einen Alternativentwurf für das eigene Leben darstellen kann. Zum anderen zeigten sie die Kämpfe und politischen Auseinandersetzungen auf, die notwendig sind, um eine nachhaltige Gesellschaft zu gestalten, sei es der Existenzkampf gegen das schwindende Wasser des Aralsees, der politische Widerstand gegen eine (weitere) Müllkippe in den Bergen oberhalb Neapels oder die Bewältigung der Entvölkerung der Sardischen Berggebiete in Folge der

Industrialisierung. Gemeinsam war allen Filmen, dass sie sehr deutlich aufzeigten, dass individuelle Entscheidungen zur eigenen Lebensführung und politische Einflussnahme dafür bestimmend sind, wie sich gesellschaftliche Verhältnisse ausgestalten.

\section{Fazit}

Die Stadt Venedig war ein eindrucksvoller Konferenzort, der aber gleichzeitig die Notwendigkeit des „Begrenzens“ deutlich zeigte. Fast schon symbolhaft erinnerte die ständige Präsenz der gigantischen Kreuzfahrtschiffe direkt neben dem Konferenzgebäude an den Massentourismus, der große Umweltprobleme für die Stadt bringt und sie in eine stets überfüllte Lage versetzt. Die Tagung selbst konnte durch die verschiedenen Themenge- biete viele WissenschaftlerInnen aber auch AktivistInnen und PraktikerInnen aus den unterschiedlichsten Bereichen zusammenbringen. Verbunden mit dem Rahmenprogramm ergaben sich so viele inter- sowie auch transdisziplinäre Ansätze und Diskussionen. Besonders auffällig waren die Lebhaftigkeit und das große Engagement, was sicherlich mit der Stärke der Degrowth-Bewegung v. a. in Südeuropa zusammenhängt. Allerdings war ein Mangel der Tagung, dass kaum die konzeptionellen Probleme von Degrowth angegangen oder die Umsetzungsprobleme angesprochen wurden. Dadurch fehlte $u$. a. die Thematisierung der kulturellen und gesellschaftlichen Schwierigkeiten des Degrowth-Konzepts, wie beispielsweise hohe Hürden bei der Akzeptanz eines Negativwachstums aufgrund von materiellen Wachstumsorientierungen sowie Verlustängsten, Ausbleiben von Belohnungseffekten oder Degrowth als Widerspruch zum ,immer wachsenden“ Gang des Lebens. Organisatorisch waren die Plenary Sessions, die häufig auf Italienisch mit Simultanübersetzung waren, zu dicht besetzt, so dass kaum Raum für Diskussionen war. Auch die ganztägigen Workshops ermöglichten zwar ein tiefes Eintauchen in ein bestimmtes Thema, allerdings konnte nur einer pro Tag besucht werden.

In Venedig trafen sich auch deutsche TeilnehmerInnen um sich bezüglich der Ausrichtung der nächsten Degrowth-Konferenz auszutauschen. Erste Gedanken und organisatorische Fragen wurden besprochen, allerdings muss sich noch zeigen, ob das Thema auch in Deutschland einen geeigneten Konferenzrahmen finden wird. 